\title{
Morfologia radicular de cultivares de café arábica submetidas a diferentes arranjos espaciais
}

\author{
Cláudio Pagotto Ronchi(1), José Márcio de Sousa Júnior(2), Wellington Luiz de Ameida(2), Daniela Silva Souza(2), \\ Natália Oliveira Silva ${ }^{(2)}$, Leandro Barbosa de Oliveira(2), Antonia Miriam Nogueira de Moura Guerra ${ }^{(3)}$ \\ e Paulo Afonso Ferreira ${ }^{(4)}$
}

\begin{abstract}
(1)Universidade Federal de Viçosa (UFV), Campus Florestal, Rodovia LMG 818, Km 06, Campus Universitário, CEP $35690-000$ Florestal, MG, Brasil. E-mail: claudiopagotto@ufv.br (2)UFV, Campus de Rio Paranaíba, Rodovia BR 354, Km 310, CEP $38810-000$ Rio Paranaíba, MG, Brasil. E-mail: josemarcio_18@hotmail.com, almeida-wl@hotmail.com, danielasouza_cp@hotmail.com, nataliasilva.13@hotmail.com, leandrosalinas2009@hotmail.com ${ }^{(3)}$ Universidade Federal do Oeste da Bahia, Campus Multidisciplinar de Barra, Rua 23 de Agosto, s/no, Assunção, CEP 47100-000 Barra, BA, Brasil. E-mail: mirianagronoma@hotmail.com (4)Universidade Federal de Mato Grosso, Campus Universitário do Araguaia, Avenida Valdon Varjão, no 6.390, CEP 78600-000 Barra do Garças, MT, Brasil. E-mail: pauloafonso@ufmt.br
\end{abstract}

Resumo - O objetivo deste trabalho foi avaliar as respostas morfológicas do sistema radicular de quatro cultivares de café arábica submetidas a diferentes arranjos espaciais. Os espaçamentos adotados foram os de $0,40,0,50,0,60,0,70$ e $0,80 \mathrm{~m}$ entre plantas na linha, tendo-se mantido fixo $3,8 \mathrm{~m}$ na entrelinha. Aos 27 , 35 e 39 meses após o transplantio, foram coletadas amostras de solo+raízes em três posições em relação aos caules das plantas e três profundidades. As raízes foram lavadas, coloridas, digitalizadas e processadas com o programa Safira. A cultivar Tupi RN IAC 1669-13 apresentou sistema radicular com características morfológicas relacionadas à absorção de nutrientes e de água superiores às das cultivares Catuaí Vermelho IAC 144, Catuaí Amarelo IAC 62 e Catuaí Amarelo IAC 32, para todos os arranjos espaciais testados. A redução no espaçamento aumenta a abundância de raízes por volume de solo, sem alterar a qualidade morfológica do sistema radicular, nem aprofundá-lo. Os sistemas radiculares das cultivares avaliadas são mais abundantes e apresentam superfície e comprimento específico maiores entre plantas adjacentes, seguidas das posições afastadas a 0,25 e $0,50 \mathrm{~m}$ do caule. A proporção de raízes na camada de $0,1 \mathrm{~m}$ de profundidade é maior que a na de $0,4 \mathrm{~m}$, para todos os espaçamentos. A variação no espaçamento entre plantas na linha altera a morfologia do sistema radicular do café arábica.

Termos para indexação: Coffea arabica, adensamento, programa Safira, sistema radicular.

\section{Root morphology of Arabica coffee cultivars subjected to different spatial arrangements}

\begin{abstract}
The objective of this work was to evaluate the morphological responses of the root system of four Arabica coffee cultivars subjected to different spatial arrangements. The adopted plant spacings were 0.40 , $0.50,0.60,0.70$, and $0.80 \mathrm{~m}$ between plants within rows, maintaining $3.8 \mathrm{~m}$ constant between rows. At 27, 35, and 39 months after transplanting, samples of soil+roots were collected at three surface positions in relation to plant stems and at three depths. Roots were washed, colored, digitalized, and processed with the Safira software. The Tupi RN IAC 1669-13 cultivar showed a root system with superior morphological traits related to the absorption of nutrients and water, in comparison to those of the Catuaí Vermelho IAC 144, Catuai Amarelo IAC 62, and Catuaí Amarelo IAC 32 cultivars, for all tested spatial arrangements. The reduction in spacing increases the amount of total root per soil volume unit, without affecting the morphological quality of the root system, nor deepening it. The root systems of the evaluated cultivars are more abundant and show higher surface and specific length between adjacent plants, followed by the 0.25 and $0.50 \mathrm{~m}$ positions away from the coffee stem. The proportion of roots at the $0.1 \mathrm{~m}$ layer is higher than at the $0.4 \mathrm{~m}$ layer, for all plant spacings. Changes in spacings between plants within lines affect the morphology of Arabica coffee root system.
\end{abstract}

Index terms: Coffea arabica, high-density planting, Safira software, root system.

\section{Introdução}

A produção de café arábica (Coffea arabica L.) na região do Cerrado brasileiro concentra-se no Estado de Minas Gerais (Fernandes et al., 2012), particularmente nas regiões do Alto Paranaíba, do Noroeste e do Triângulo Mineiro, onde estima-se uma produção de 5,004 milhões de sacas para a safra 2015 (Acompanhamento da safra brasileira, 2015). Nessa região, a implantação das lavouras ocorre em 
filas ou renques abertos para permitir o cultivo e a colheita mecanizados, o que também facilita os tratos culturais (Matiello et al., 2010; Fernandes et al., 2012). O espaçamento comumente praticado é o de 4,0x0,5 $\mathrm{m}$, para as cultivares de porte baixo, como o Catuaí, que são amplamente cultivadas no Cerrado (Santinato et al., 2008; Matiello et al., 2010).

Embora a definição do melhor espaçamento de plantio dependa de diversas variáveis, como cultivares, porte da planta, topografia e condições de manejo, o arranjo ou a melhor distribuição das plantas dentro das fileiras, tendendo à redução no espaçamento entre plantas, pode representar aumentos bastante significativos em produtividade por área (Santinato et al., 2008; Matiello et al., 2010). De fato, em trabalho recente, Andrade et al. (2014) observaram que combinações mais produtivas, para uma mesma população de plantas por hectare, ocorreram quando se empregou menor espaçamento entre plantas na linha.

A redução no espaçamento de plantio geralmente aumenta a produtividade por área, sobretudo nas primeiras colheitas (Braccini et al., 2005; Paulo et al., 2005; Pereira et al., 2011; Andrade et al., 2014), o que pode reduzir o gasto com fertilizantes (Guarçoni M., 2011) em até 57\% (Figueiredo et al., 2006). Essa utilização mais eficiente de fertilizantes decorre de alterações positivas na fertilidade dos solos e na ciclagem de nutrientes em sistemas adensados (Pavan et al., 1999; Augusto et al., 2007; Bragança et al., 2009; Guarçoni M., 2011). Além disso, Cassidy \& Kumar (1984) demonstraram que, em altas densidades de plantio, a exploração do solo pelas raízes do cafeeiro é mais completa, o que possibilita a utilização mais eficiente de água e minerais, em camadas superficiais ou profundas do solo.

Assim, as alterações no sistema radicular do cafeeiro parecem explicar o seu comportamento em cultivo adensado. Sabe-se que o sistema radicular do cafeeiro apresenta alta plasticidade, e que sua distribuição e morfologia são dependentes da idade da planta (Bragança, 2005), do tipo de irrigação e do manejo nutricional adotados (Barreto et al., 2006), da relação entre fonte e dreno (Alves et al., 2011), do método de formação de mudas (Partelli et al., 2006, 2014; Silva et al., 2010), das características químicas do solo (Mota et al., 2006), da calagem em subsuperfície (Rodrigues et al., 2001), das práticas culturais (Rena \& DaMatta, 2002), da competição e das espécies de plantas daninhas (Ronchi et al., 2007), entre outros (Rena \& DaMatta, 2002; DaMatta et al., 2007).

No entanto, as alterações do sistema radicular do café arábica, à medida que se alteram as densidades de plantio, são escassas e resumem-se basicamente ao trabalho de Cassidy \& Kumar (1984), citado anteriormente, realizado em Zimbabwe, na África. No caso do comportamento do sistema radicular, em função dos diferentes materiais genéticos, os principais trabalhos foram feitos com C. canephora (Alfonsi et al., 2005; Bragança, 2005; Pinheiro et al., 2005; Silva et al., 2010; Covre et al., 2013); porém, não há informações para $C$. arabica, que atualmente tem muitas cultivares novas (Carvalho, 2008; Bliska et al., 2011).

O objetivo deste trabalho foi avaliar as respostas morfológicas do sistema radicular de quatro cultivares de café arábica submetidas a diferentes arranjos espaciais.

\section{Material e Métodos}

A área experimental está localizada na Fazenda Transagro S.A., em Rio Paranaíba, MG (19¹3'1"S, $46^{\circ} 20^{\prime} 36^{\prime \prime} \mathrm{W}$, a $906 \mathrm{~m}$ de altitude), pertencente à região do Alto Paranaíba (Cerrado mineiro). Esta região é caracterizada por um verão quente e chuvoso, seguido de inverno seco e frio (Fernandes et al., 2012). O solo da área experimental foi classificado como Latossolo Vermelho-Amarelo distrófico, de textura argilosa e relevo suave ondulado (Motta et al., 2004; Andrade et al., 2011). A análise química do solo indicou, na camada de $0-20 \mathrm{~cm}$, os seguintes atributos químicos: pH 5,83; 33,7 $\mathrm{g} \mathrm{kg}^{-1}$ de matéria orgânica; $\mathrm{V}$ de 46,5\%; saturação por alumínio $(\mathrm{m})$ de 2,5\%; $0,84 \mathrm{mg} \mathrm{dm}^{-3} \mathrm{de}$ $\mathrm{P} ; 250 \mathrm{mg} \mathrm{dm}^{-3} \mathrm{de} \mathrm{K} ; 1,60 \mathrm{cmol}_{\mathrm{c}} \mathrm{dm}^{-3} \mathrm{de} \mathrm{Ca} ; 0,47 \mathrm{cmol}_{\mathrm{c}}$ $\mathrm{dm}^{-3}$ de $\mathrm{Mg}$; e CTC (T) de 5,70 $\mathrm{cmol}_{\mathrm{c}} \mathrm{dm}^{-3}$.

O experimento foi iniciado em janeiro de 2010, com a implantação de uma lavoura comercial formada por quatro cultivares de café arábica. As mudas com quatro pares de folhas foram transplantadas para os sulcos, de acordo com o método de plantio comumente utilizado na cafeicultura do Cerrado (Santinato et al., 2008), no espaçamento de $3,8 \mathrm{~m}$ na entrelinha. Os tratos culturais, durante o ano agrícola, consistiram basicamente de manejo de plantas daninhas com herbicidas, roçadeiras ou trincha, manejo fitossanitário, calagem na linha de plantio e aplicação de água e fertilizantes (fertirrigação) 
por um sistema de gotejamento, igualmente em toda a área experimental. $\mathrm{O}$ manejo da irrigação foi feito de setembro a maio, e o sistema de irrigação era ligado 8 horas por dia, sempre que a água facilmente disponível inicial (AFDI) atingia o valor de 50\%, elevando-a para, no mínimo, 80\%. Durante o ano agrícola 2012/2013, foram aplicados 407, 162 e $387 \mathrm{~kg} \mathrm{ha}^{-1}$ de $\mathrm{N}, \mathrm{P}_{2} \mathrm{O}_{5}$ e $\mathrm{K}_{2} \mathrm{O}$, respectivamente, além de $677 \mathrm{~kg} \mathrm{ha}^{-1}$ de calcário dolomítico e $1.531 \mathrm{~kg} \mathrm{ha}^{-1}$ de esterco de galinha.

Utilizou-se o delineamento de blocos ao acaso com os tratamentos dispostos em arranjo fatorial com quatro repetições. Foram avaliadas quatro cultivares de café arábica: Catuaí Vermelho IAC 144, Catuaí Amarelo IAC 62, Catuaí Amarelo IAC 32 e Tupi RN IAC 1669-13, escolhidas por serem materiais amplamente empregados na cafeicultura mecanizada (Catuaí Vermelho IAC 144) ou de grande potencial para uso no Cerrado, por apresentarem coloração amarela dos frutos e alta adaptabilidade (Catuaí Amarelo IAC 62 e Catuaí Amarelo IAC 32), alta qualidade de bebida, resistência à ferrugem e a nematoides, e adaptação para cultivo adensado (Tupi RN IAC 1669-13) (Carvalho, 2008). Cada cultivar foi implantada em cinco espaçamentos entre plantas na linha $(0,4,0,5,0,6,0,7$ e $0,8 \mathrm{~m})$. Os blocos foram dispostos na linha de plantio, com dez plantas por unidade experimental.

As amostragens de raízes foram realizadas num interstício de 12 meses, em três épocas distintas: abril de 2012, aos 27 meses após o transplantio; novembro de 2012, aos 35 meses após o transplantio; e em abril de 2013, aos 39 meses após o transplantio. Em cada época de coleta, em todas as cultivares, as amostragens de raízes foram realizadas em três posições sob a copa do cafeeiro: posição 1, entre duas plantas adjacentes, no mesmo alinhamento dos caules na linha de plantio, na "metade" do espaçamento entre plantas, ao se considerar que o espaçamento entre plantas variou de acordo com o tratamento; posições 2 e 3 , afastadas a 0,25 e $0,50 \mathrm{~m}$ do caule, respectivamente, no sentido perpendicular à linha de plantio. Em cada posição de coleta, amostras de solo foram retiradas em três profundidades: $0,10,0,20$ e $0,40 \mathrm{~m}$, como descrito a seguir.

Escavou-se com uma cavadeira até à profundidade desejada, e, em seguida, introduziu-se o cilindro do trado Uhland, para coleta de uma amostra de solo+raízes de $270 \mathrm{~cm}^{3}$. As amostras foram acondicionadas em sacos de plástico, levadas para o laboratório e armazenadas em geladeira até o processamento. Posteriormente, foram lavadas em água corrente, sobre peneiras de malha inferior a $1,0 \mathrm{~mm}$, coloridas com violeta genciana (2\%) por 5 min e dispostas em papel toalha para retirada do excesso de corante. Em seguida, as raízes foram organizadas sobre uma folha de transparência (filme de poliestireno transparente), sem sobreposição. Para produzir um contraste de fundo, foi colocada uma folha de papel branca sobre o conjunto raízes +transparência; todo o conjunto foi escaneado em escala de cinza, com resolução de 200 dpi. As amostras de raízes foram secas em estufa de circulação forçada de ar a $70^{\circ} \mathrm{C}$, até massa constante, para quantificação da matéria seca.

As imagens digitalizadas foram processadas individualmente com a utilização do programa Safira, desenvolvido pela Embrapa (Jorge \& Rodrigues, 2008), com limite de limiarização definido em 150 (parâmetro inerente ao programa). A partir dos resultados obtidos pelo programa, foram estimados os seguintes parâmetros fisiológicos relacionados à morfologia do sistema radicular: massa de matéria seca total de raízes por volume de solo (MST), em $\mathrm{mg} \mathrm{cm}$; comprimento total de raízes por volume de solo (CT), em $\mathrm{cm} \mathrm{cm}^{-3}$; volume total de raízes por volume de solo (VT), em $\mathrm{mm}^{3} \mathrm{~cm}^{-3}$; área total de raízes por volume de solo (AT), em $\mathrm{mm}^{2} \mathrm{~cm}^{-3}$; superfície específica de raízes (SE), obtida pela relação entre AT e MST, em m $\mathrm{kg}^{-1}$; comprimento específico de raízes, obtido pela relação entre CT e MST, em m g${ }^{-1}$; e diâmetro médio das raízes (DM), em milímetros.

Os dados médios obtidos nas três épocas foram submetidos à análise de variância, pelo teste $\mathrm{F}$, tendo-se utilizado arranjo fatorial $4 \times 5 \times 3 \times 3$ (quatro cultivares, cinco espaçamentos, três posições sobre a copa e três profundidades de coleta). Apenas as interações de interesse, relacionadas ao fator espaçamento, foram desdobradas, tendo-se utilizado o teste de Tukey, a 5\% de probabilidade, para comparação entre as médias dos tratamentos. Ajustaram-se equações de regressão ao se relacionar cada variável ao espaçamento entre plantas na linha, tendo-se optado por modelos significativos, com alto $\mathrm{R}^{2}$ e com lógica biológica.

\section{Resultados e Discussão}

A cultivar Tupi RN IAC 1669-13 destacou-se entre as cultivares avaliadas. Em comparação, por

Pesq. agropec. bras., Brasília, v.50, n.3, p.187-195, mar. 2015 DOI: 10.1590/S0100-204X2015000300001 
exemplo, à cultivar tradicional Catuaí Vermelho IAC 144 apresentou MST, CT, VT, AT, SE e CE significativamente superiores em 24, 78, 341, 49, 18 e $15 \%$, respectivamente, independentemente dos outros fatores estudados (Tabela 1). O DM das raízes $(0,315 \mathrm{~mm})$ foi, em média, 3,8\% inferior $(\mathrm{p}<0,05)$ ao das cultivares Catuaí Vermelho IAC 144 e Catuaí Amarelo IAC 32, mas significativamente igual ao da Catuaí Amarelo IAC $62(0,318 \mathrm{~mm})$.

Numa análise geral, os resultados obtidos são indicativos de que a cultivar Tupi RN IAC 1669-13 apresentou características morfológicas relacionadas à absorção de água e nutrientes quantitativa e qualitativamente superiores às das demais cultivares. Isso pode explicar seu elevado desempenho agronômico, sobretudo no Cerrado mineiro, onde tem sido amplamente cultivada, por ser resistente à ferrugem e ao nematoide Meloidogyne exigua (Carvalho, 2008; Bliska et al., 2011).

Independentemente das cultivares, da posição e da profundidade de coleta, observou-se aumento, principalmente em MST, VT e AT, à medida que houve redução no espaçamento de 0,8 para $0,4 \mathrm{~m}$ entre plantas na linha (Figura 1 A, C e D), sem afetar a SE e o CE (Figura 1 E e F). Os padrões de redução (modelos matemáticos) alteraram-se em função de cada variável (Figura 1). Esses resultados são indicativos de que o aumento da população de plantas por hectare, de acordo com o adensamento na linha de plantio, promove, de forma geral, maior abundância ou distribuição quantitativa de raízes por unidade de volume de solo, sem efeitos positivos na qualidade das raízes, que seriam expressos pelo aumento em SE e CE.

Essas evidências têm grande importância científica, pois podem contribuir de fato para explicar o melhor aproveitamento de fertilizantes em plantios mais adensados da cafeicultura moderna, como mostrado há 30 anos com plantas em covas, em Zimbabwe, África (Cassidy \& Kumar, 1984). Atualmente, diversos artigos científicos (Augusto et al., 2007; Bragança et al., 2009; Guarçoni M., 2011) citam o trabalho de Pavan et al. (1994) ao atribuir o melhor aproveitamento de fertilizantes em plantios adensados a um sistema radicular com maior densidade. Entretanto, apesar de ter sido uma hipótese oportunamente levantada, cabe ressaltar que Pavan et al. (1994) não amostraram o sistema radicular.

Os resultados obtidos no presente trabalho corroboram os argumentos de Guarçoni (2011), de que o aumento na densidade de plantas não precisa necessariamente ser acompanhado de um aumento proporcional na aplicação de fertilizantes por área. Isso porque os maiores valores obtidos para matéria seca, área superficial e volume de raízes por volume de solo, em cafeeiros adensados, podem compensar um menor input de fertilizantes, como observado no

Tabela 1. Médias gerais das variáveis matéria seca total (MST), comprimento total (CT), volume total (VT), área total (AT), superfície específica (SE), comprimento específico (CE) e diâmetro médio (DM) de raízes de plantas de café arábica (Coffea arabica), de acordo com cultivares, posição e profundidade de coleta ${ }^{(1)}$.

\begin{tabular}{|c|c|c|c|c|c|c|c|}
\hline Variável & $\begin{array}{c}\mathrm{MST} \\
\left(\mathrm{mg} \mathrm{cm}^{-3}\right)\end{array}$ & $\begin{array}{c}\mathrm{CT} \\
\left(\mathrm{cm} \mathrm{cm}^{-3}\right)\end{array}$ & $\begin{array}{c}\mathrm{VT} \\
\left(\mathrm{mm}^{3} \mathrm{~cm}^{-3}\right)\end{array}$ & $\begin{array}{c}\mathrm{AT} \\
\left(\mathrm{mm}^{2} \mathrm{~cm}^{-3}\right)\end{array}$ & $\begin{array}{c}\mathrm{SE} \\
\left(\mathrm{m}^{2} \mathrm{~kg}^{-1}\right)\end{array}$ & $\begin{array}{c}\mathrm{CE} \\
\left(\mathrm{cm} \mathrm{g}^{-1}\right)\end{array}$ & $\begin{array}{c}\mathrm{DM} \\
(\mathrm{mm})\end{array}$ \\
\hline \multicolumn{8}{|l|}{ Cultivar } \\
\hline Catuaí Vermelho IAC 144 & $0,045 b$ & $11,50 \mathrm{~b}$ & $2,45 b$ & $1,42 b c$ & $31,66 b$ & $29,39 b$ & $0,328 \mathrm{a}$ \\
\hline Catuaí Amarelo IAC 62 & $0,047 \mathrm{~b}$ & $9,44 \mathrm{c}$ & $1,89 \mathrm{c}$ & $1,32 \mathrm{c}$ & $29,82 \mathrm{c}$ & $22,61 d$ & $0,318 b$ \\
\hline Catuaí Amarelo IAC 32 & $0,054 \mathrm{a}$ & $12,12 b$ & $2,75 b$ & $1,53 b$ & $29,56 \mathrm{c}$ & $24,87 \mathrm{c}$ & $0,327 \mathrm{a}$ \\
\hline Tupi RN IAC 1699-13 & $0,056 \mathrm{a}$ & $20,43 a$ & $10,81 \mathrm{a}$ & $2,12 \mathrm{a}$ & $37,44 \mathrm{a}$ & $33,91 \mathrm{a}$ & $0,315 b$ \\
\hline \multicolumn{8}{|c|}{ Posição sob a copa do cafeeiro(2) } \\
\hline Posição 1 & $0,054 \mathrm{a}$ & $15,92 \mathrm{a}$ & $5,60 \mathrm{a}$ & $1,72 \mathrm{a}$ & $32,62 \mathrm{a}$ & $28,78 \mathrm{a}$ & $0,321 \mathrm{a}$ \\
\hline Posição 2 & $0,051 \mathrm{a}$ & $12,86 \mathrm{~b}$ & $4,26 b$ & $1,64 b$ & $32,64 b$ & $28,17 \mathrm{a}$ & $0,322 \mathrm{a}$ \\
\hline Posição 3 & $0,046 b$ & $11,34 \mathrm{c}$ & $3,57 \mathrm{c}$ & $1,42 b$ & $31,10 \mathrm{~b}$ & $26,14 b$ & $0,323 a$ \\
\hline \multicolumn{8}{|l|}{ Profundidade } \\
\hline $0,1 \mathrm{~m}$ & $0,051 \mathrm{a}$ & $14,80 \mathrm{a}$ & $5,44 \mathrm{a}$ & $1,68 \mathrm{a}$ & $32,82 \mathrm{a}$ & $27,65 b$ & $0,324 a$ \\
\hline $0,2 \mathrm{~m}$ & $0,054 \mathrm{a}$ & $13,16 b$ & $4,09 b$ & $1,67 \mathrm{a}$ & $31,23 b$ & $26,18 \mathrm{c}$ & $0,325 \mathrm{a}$ \\
\hline $0,4 \mathrm{~m}$ & $0,045 b$ & $12,15 \mathrm{c}$ & $3,91 \mathrm{c}$ & $1,43 b$ & $32,31 \mathrm{ab}$ & $29,26 \mathrm{a}$ & $0,318 b$ \\
\hline
\end{tabular}

${ }^{(1)}$ Médias seguidas de letras iguais, para cada variável, nas colunas, não diferem pelo teste de Tukey, a $5 \%$ de probabilidade. (2)Posição 1 , entre duas plantas adjacentes, no mesmo alinhamento dos caules na linha de plantio, na "metade" do espaçamento entre plantas, ao se considerar que o espaçamento entre plantas variou de acordo com o tratamento; e posições 2 e 3, afastadas a 0,25 e $0,50 \mathrm{~m}$ do caule, respectivamente, no sentido perpendicular à linha de plantio. 
presente trabalho. Nesse contexto, Figueiredo et al. (2006) relataram a possibilidade de redução nas doses de $\mathrm{N}, \mathrm{P}_{2} \mathrm{O}_{5}$ e $\mathrm{K}_{2} \mathrm{O}$ em 57, 90 e $52 \%$, respectivamente, para cafeeiros adensados.

O sistema radicular do cafeeiro arábica apresenta extensão lateral máxima de 1,0 m (Rena \& Guimarães, 2000), sendo que aproximadamente $75 \%$ concentramse na projeção da copa (Mota et al., 2006). Para $C$. canephora 'Apoatã', Barreto et al. (2006) constataram que a distância efetiva do sistema radicular não ultrapassou os $0,8 \mathrm{~m}$ do tronco. No presente trabalho, verificou-se que o CT e o VT foram superiores na posição 1 em relação à posição 2 , a qual foi superior à posição 3; no caso da MST e do $\mathrm{CE}$, não houve diferença significativa $(\mathrm{p}>0,05)$ entre as posições $1 \mathrm{e}$ 2 , mas estas foram significativamente superiores em relação à posição 3. Já a $\mathrm{AT}$ e a $\mathrm{SE}$ foram superiores na posição 1 , quando comparada às demais, entre as quais não houve diferença. Por fim, o DM de raízes foi significativamente semelhante, independentemente da posição de coleta (Tabela 1).

Desse modo, independentemente dos demais fatores avaliados, concluiu-se que, a maior extensão do sistema radicular e, também, a sua qualidade ocorrem nas imediações do ramo ortotrópico, como relatado por Mota et al. (2006). Por se tratar de uma lavoura jovem, o sistema radicular expande-se com o aumento da idade (Bragança, 2005), o que pode ser resultado da presença do sistema de fertirrigação nessa posição. Partelli et al. (2014) também constataram que, em lavouras de $C$. canephora, a concentração de raízes finas ocorreu distante do ramo ortotrópico, onde as adubações de solo eram feitas. Contudo, esse padrão de resposta pode não ser um consenso, haja vista que Barreto et al. (2006) demonstraram que, em lavouras fertirrigadas, a densidade de raízes foi significativamente superior em pontos mais distantes do bulbo úmido, mas sob a copa.

Não houve diferenças significativas $(p>0,05)$ entre as profundidades de 0,1 e $0,2 \mathrm{~m}$, para MST e AT; porém, estas variáveis foram superiores $(\mathrm{p}<0,05)$, em média, 16,7 e 17,1\%, respectivamente, à profundidade de $0,4 \mathrm{~m}$. O CT e o VT reduziram-se significativamente à medida que as coletas foram feitas em maior profundidade no perfil do solo; e o CE foi superior na maior profundidade, provavelmente pela redução não proporcional entre MST e CT, o que é confirmado pelo menor diâmetro médio de raízes $(0,318 \mathrm{~mm})$ na profundidade de $0,4 \mathrm{~m}$, em comparação às demais
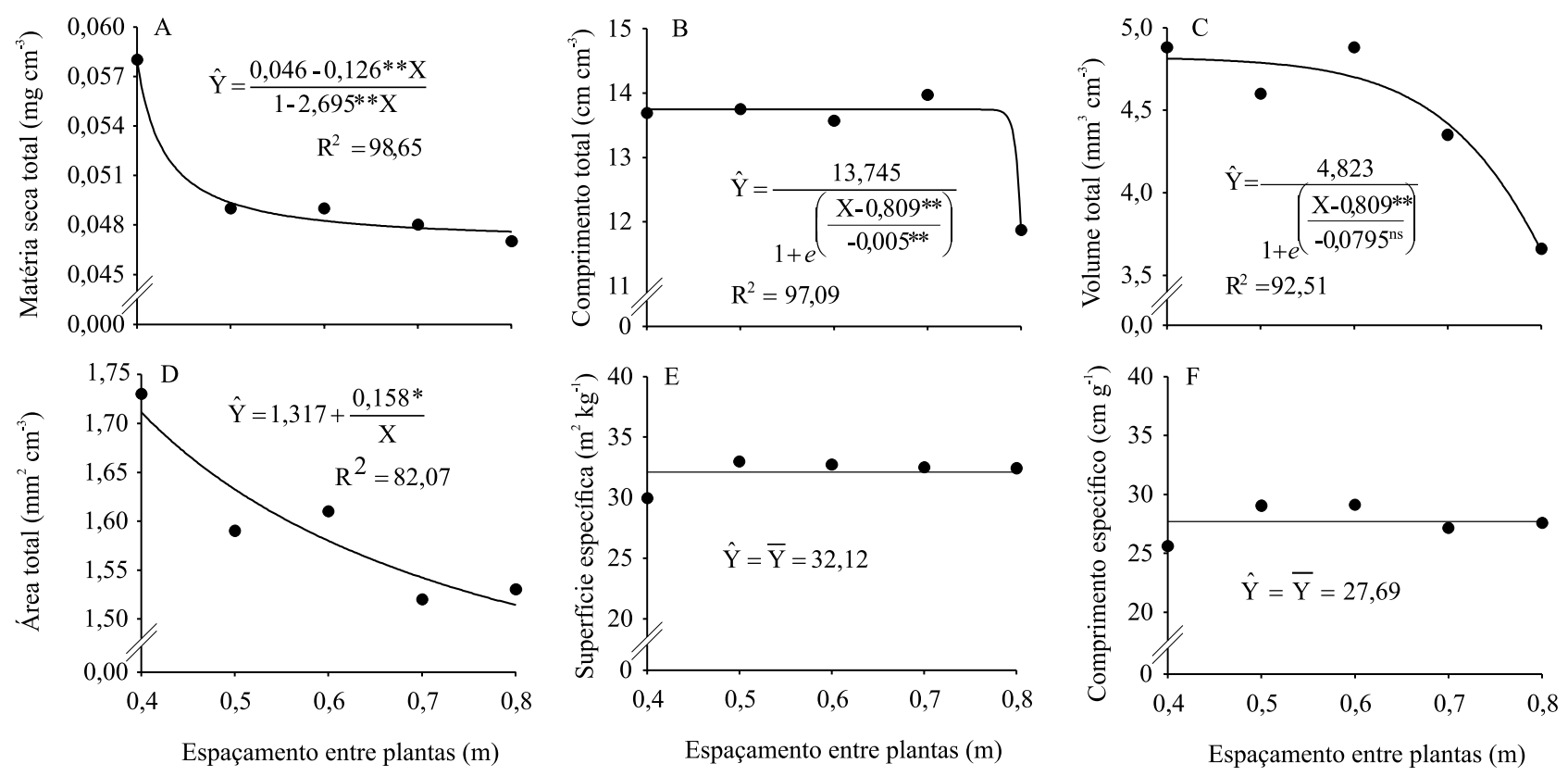

Figura 1. Efeito do espaçamento entre plantas sobre as variáveis: massa de matéria seca total (A), comprimento total (B), volume total (C), área total (D), superfície específica (E) e comprimento específico (F) de raízes de plantas de café arábica (Coffea arabica), independentemente de cultivar, posição sob a copa do cafeeiro e profundidade de coleta. * $\mathrm{e}^{* *}$ Significativo

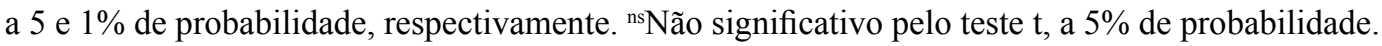


( 0,325 mm) (Tabela 1). Partelli et al. (2014), ao avaliar o café 'Conilon', também observaram que a área superficial, o volume e o comprimento total de raízes foram reduzidos com o aprofundamento no perfil do solo, assim como o diâmetro médio de raízes, em razão do empobrecimento da fertilidade do solo.

Conforme Rena \& Guimarães (2000), o sistema radicular do cafeeiro arábica apresenta profundidade média de $1,2 \mathrm{~m}$, sendo que mais de $80 \%$ se concentra nos primeiros $0,5 \mathrm{~m}$. Mota et al. (2006) encontraram $63 \%$ do sistema radicular absorvente até $0,2 \mathrm{~m}$ de profundidade. O maior volume do sistema radicular nas camadas superiores é explicado pela presença de cobertura morta e pelo sombreamento (Cassidy \& Kumar, 1984), pela ausência de uma raiz pivotante proeminente e pelas adubações de solo na superfície do solo (Rena \& Guimarães, 2000). Dessa forma, também é possível atribuir a maior concentração de raízes nessas camadas, no presente trabalho, à distribuição de fertilizantes de modo concentrado via fertirrigação. Essa conclusão é razoável, ao se considerar que a profundidade efetiva do sistema radicular se altera com a adoção da fertirrigação, com o posicionamento e o espaçamento entre os gotejadores (Barreto et al., 2006).

Ocorreram interações significativas entre o espaçamento e as cultivares, para as variáveis CT, VT, $\mathrm{SE}, \mathrm{CE}$ e DM; as posições de coleta, para CT, VT, SE,
AT e CE; e as profundidades de coleta, para CT e CE. O CT foi maior na cultivar Tupi RN IAC 1669-13, em comparação à Catuaí Vermelho IAC 144, para todos os espaçamentos testados, com destaque para o espaçamento de $0,8 \mathrm{~m}$, que foi superior em $111 \%$ $(\mathrm{p}<0,05)$ (Tabela 2). Esse atributo morfofisiológico mostra o alto potencial da cultivar Tupi RN IAC 1669-13 em absorver água e nutrientes (Cassidy \& Kumar, 1984; Rena \& Guimarães, 2000; Rena \& DaMatta, 2002) e em se adaptar a diferentes arranjos espaciais, com indicação, inclusive, para cultivos adensados (Bliska et al., 2011).

O CT foi superior na posição 1 , nos espaçamentos de 0,5 a 0,8 m, mas não no de 0,4 m, em comparação às demais posições (Tabela 2), o que pode ser explicado pela intersecção de sistemas radiculares de plantas adjacentes na linha de plantio (Rena \& Guimarães, 2000) e pelo fato de o menor espaçamento forçar o crescimento lateral das raízes, por falta de espaço na linha. Ao se comparar as posições 2 e 3, observouse que, à medida que se distanciou do caule, no sentido perpendicular à linha de plantio, o tamanho do sistema radicular avaliado pelo CT uniformizouse lateralmente em espaçamentos mais amplos (0,7 e $0,8 \mathrm{~m}$ vs. 0,5 e $0,6 \mathrm{~m})$. Em $C$. canephora, o sistema radicular apresentou-se muito homogêneo lateralmente, independentemente da profundidade

Tabela 2. Desdobramento das interações significativas entre espaçamento e cultivares, espaçamento e posição, e espaçamento e profundidade de coleta, independentemente dos demais fatores avaliados, para a variável comprimento total de raízes por volume de solo (CT, $\mathrm{cm} \mathrm{cm}^{-3}$ ), em plantas de café arábica (Coffea arabica) ${ }^{(1)}$.

\begin{tabular}{|c|c|c|c|c|c|}
\hline \multirow[t]{2}{*}{ Variável } & \multicolumn{5}{|c|}{ Espaçamentos entre plantas na linha (m) } \\
\hline & 0,4 & 0,5 & 0,6 & 0,7 & 0,8 \\
\hline Cultivar & \multicolumn{5}{|c|}{ Interação espaçamento vs. cultivares } \\
\hline Catuaí Vermelho IAC 144 & $13,01 \mathrm{bA}$ & $12,07 \mathrm{bA}$ & $10,91 \mathrm{bcA}$ & $12,82 \mathrm{bA}$ & $8,70 \mathrm{cB}$ \\
\hline Catuaí Amarelo IAC 62 & $8,05 \mathrm{cB}$ & $10,77 \mathrm{bA}$ & $9,75 \mathrm{cAB}$ & $9,70 \mathrm{cAB}$ & $8,95 \mathrm{cAB}$ \\
\hline Catuaí Amarelo IAC 32 & $12,51 \mathrm{bA}$ & $12,63 \mathrm{bA}$ & $12,69 \mathrm{bA}$ & $11,32 \mathrm{bcA}$ & $11,46 \mathrm{bA}$ \\
\hline Tupi RN IAC 16699-13 & $21,22 \mathrm{aAB}$ & $19,54 \mathrm{aBC}$ & $20,95 \mathrm{aAB}$ & $22,06 \mathrm{aA}$ & $18,40 \mathrm{aC}$ \\
\hline Posição sob a copa do cafeeiro(2) & \multicolumn{5}{|c|}{ Interação espaçamento vs. posição de coleta } \\
\hline Posição 1 & $14,87 \mathrm{aBC}$ & $16,25 \mathrm{aAB}$ & $17,70 \mathrm{aA}$ & $17,10 \mathrm{aA}$ & $13,69 \mathrm{aC}$ \\
\hline Posição 2 & $13,54 \mathrm{abAB}$ & $14,25 \mathrm{bA}$ & $12,93 \mathrm{bABC}$ & $12,39 \mathrm{bBC}$ & $11,21 b C$ \\
\hline Posição 3 & $12,68 \mathrm{bA}$ & $10,75 \mathrm{cBC}$ & $10,11 \mathrm{cC}$ & $12,43 \mathrm{bAB}$ & $10,73 \mathrm{bBC}$ \\
\hline Profundidade & \multicolumn{5}{|c|}{ Interação espaçamento vs. profundidade de coleta } \\
\hline $0,10 \mathrm{~m}$ & $15,91 \mathrm{aA}$ & $15,16 \mathrm{aA}$ & $14,33 \mathrm{aAB}$ & $16,02 \mathrm{aA}$ & $12,61 \mathrm{aB}$ \\
\hline $0,20 \mathrm{~m}$ & $13,47 \mathrm{bAB}$ & $12,83 \mathrm{bAB}$ & $14,27 \mathrm{aA}$ & $13,03 \mathrm{bAB}$ & $12,21 \mathrm{abB}$ \\
\hline $0,40 \mathrm{~m}$ & $11,71 \mathrm{cAB}$ & $13,26 \mathrm{bA}$ & $12,14 \mathrm{bAB}$ & $12,87 \mathrm{bA}$ & $10,81 \mathrm{bB}$ \\
\hline
\end{tabular}

${ }^{(1)}$ Médias seguidas de letras iguais, minúsculas nas colunas e maiúsculas nas linhas, para cada interação, não diferem pelo teste de Tukey, a 5\% de probabilidade. ${ }^{(2)}$ Posição 1, entre duas plantas adjacentes, no mesmo alinhamento dos caules na linha de plantio, na "metade" do espaçamento entre plantas, ao se considerar que o espaçamento entre plantas variou de acordo com o tratamento; e posições 2 e 3 , afastadas a 0,25 e 0,50 m do caule, respectivamente, no sentido perpendicular à linha de plantio. 
amostrada (Partelli et al., 2014). Quanto ao CE, foi superior na posição 1 , em comparação às posições 2 e 3, entre as quais não houve diferença significativa, observada apenas nos espaçamentos mais amplos, de 0,7 e $0,8 \mathrm{~m}$ (Tabela 3 ).

Verificou-se que o CT foi 15,3 e $26,4 \%$ menor $(\mathrm{p}<0,05)$ a 0,2 e $0,4 \mathrm{~m}$, respectivamente, que a $0,10 \mathrm{~m}$ de profundidade (Tabela 2), somente para o espaçamento de 0,4 m entre plantas. Em espaçamentos mais amplos, de 0,5 a $0,8 \mathrm{~m}$, a distribuição das raízes nas primeiras camadas foi mais homogênea, de forma que diferenças significativas ocorreram entre a camada mais superficial $(0,10 \mathrm{~m})$ e a mais profunda $(0,4 \mathrm{~m})$, com redução média de 15,6\% no CT. Esses resultados diferem ligeiramente dos de Cassidy \& Kumar (1984), que encontraram maior densidade de raízes em profundidade, com redução no espaçamento. Quando se comparou o CT (Tabela 2) entre os espaçamentos, dentro de uma mesma profundidade, constatou-se um padrão não muito consistente de menor abundância de raízes no espaçamento de $0,8 \mathrm{~m}$, em relação ao de $0,4 \mathrm{~m}$, principalmente na camada de $0,1 \mathrm{~m}$.

Por se tratar de uma lavoura em franco desenvolvimento, o sistema radicular nas camadas mais profundas $(0,4 \mathrm{~m})$ está em pleno crescimento. Esse resultado é semelhante aos obtidos por Silva et al. (2009), para a cultivar Catuaí Vermelho IAC 144, com 3 a 5 anos de idade, cultivada sem irrigação no espaçamento de $1,75 \times 0,70 \mathrm{~m}$. De acordo com esses autores, a maior parte do sistema radicular (58\%) está distribuída na camada superficial de solo $(0-0,3 \mathrm{~m})$ e menos de $10 \%$ atinge profundidades maiores que $1,0 \mathrm{~m}$; além disso, as raízes existentes na camada superficial (0-0,3 m) são as mais importantes na extração de água em planta de café, o que corrobora a definição clássica de raízes absorventes, com diâmetro inferior a 1,0 mm (Rena \& DaMatta, 2002), que foi a classe de raízes avaliada no presente trabalho.

Conhecimentos sobre a morfologia e a distribuição do sistema radicular foram de grande importância para explicar o crescimento da copa e a produção do cafeeiro (Pereira et al., 2011), a eficiência na produção de raízes por unidade de nutriente absorvido (Amaral et al., 2011), a estimativa da necessidade de água (Silva et al., 2009), bem como para definir o sistema de irrigação (Barreto et al., 2006). Espera-se, portanto, que as informações morfofisiológicas do sistema radicular do café arábica apresentadas e discutidas, sobretudo de cultivares recentemente desenvolvidas, contribuam para o entendimento das respostas do cafeeiro ao adensamento, que é uma tendência na cafeicultura moderna, segundo Matiello et al. (2010).

Tabela 3. Desdobramento das interações significativas entre espaçamento e cultivares, espaçamento e posição, e espaçamento e profundidade de coleta, independentemente dos demais fatores avaliados, para a variável comprimento específico de raízes $\left(\mathrm{CE}, \mathrm{cm} \mathrm{g}^{-1}\right)$, em plantas de café arábica (Coffea arabica) $)^{(1)}$.

\begin{tabular}{|c|c|c|c|c|c|}
\hline \multirow[t]{2}{*}{ Variável } & \multicolumn{5}{|c|}{ Espaçamentos entre plantas na linha (m) } \\
\hline & 0,4 & 0,5 & 0,6 & 0,7 & 0,8 \\
\hline Cultivar & \multicolumn{5}{|c|}{ Interação espaçamento vs. cultivares } \\
\hline Catuaí Vermelho IAC 144 & $23,18 \mathrm{bC}$ & $34,77 \mathrm{aA}$ & $28,18 \mathrm{bB}$ & $28,55 \mathrm{bB}$ & $32,29 \mathrm{aA}$ \\
\hline Catuaí Amarelo IAC 62 & $20,69 \mathrm{bB}$ & $21,97 \mathrm{~dB}$ & $25,98 \mathrm{bA}$ & $23,76 \mathrm{cAB}$ & $20,67 \mathrm{cB}$ \\
\hline Catuaí Amarelo IAC 32 & $21,44 \mathrm{bB}$ & $27,86 \mathrm{cA}$ & $26,15 \mathrm{bA}$ & $22,44 \mathrm{cB}$ & $26,50 \mathrm{bA}$ \\
\hline Tupi RN IAC 16699-13 & $37,12 \mathrm{aA}$ & $31,57 \mathrm{bB}$ & $36,16 \mathrm{aA}$ & $33,84 \mathrm{aAB}$ & $30,90 \mathrm{aB}$ \\
\hline Posição sob a copa do cafeeiro ${ }^{(2)}$ & \multicolumn{5}{|c|}{ Interação espaçamento vs. posição de coleta } \\
\hline Posição 1 & $27,52 \mathrm{aA}$ & $28,64 \mathrm{abA}$ & $28,85 \mathrm{bA}$ & $29,79 \mathrm{aA}$ & $29,11 \mathrm{aA}$ \\
\hline Posição 2 & $26,11 \mathrm{aBC}$ & $30,53 \mathrm{aA}$ & $31,93 \mathrm{aA}$ & $24,73 \mathrm{bC}$ & $27,60 \mathrm{bB}$ \\
\hline Posição 3 & $23,20 \mathrm{bB}$ & $27,95 \mathrm{bA}$ & $26,57 \mathrm{bA}$ & $26,93 \mathrm{bA}$ & $26,06 \mathrm{bA}$ \\
\hline Profundidade & \multicolumn{5}{|c|}{ Interação espaçamento vs. profundidade de coleta } \\
\hline $0,10 \mathrm{~m}$ & $25,16 \mathrm{aC}$ & $29,02 \mathrm{bAB}$ & $27,84 \mathrm{bABC}$ & $30,03 \mathrm{aA}$ & $26,24 \mathrm{bBC}$ \\
\hline $0,20 \mathrm{~m}$ & $26,10 \mathrm{aB}$ & $26,36 \mathrm{cB}$ & $25,13 \mathrm{cB}$ & $23,76 \mathrm{bB}$ & $29,55 \mathrm{aA}$ \\
\hline $0,40 \mathrm{~m}$ & $25,56 \mathrm{aB}$ & $31,74 \mathrm{aA}$ & $34,38 \mathrm{aA}$ & $27,65 \mathrm{aB}$ & $26,98 \mathrm{bB}$ \\
\hline
\end{tabular}

${ }^{(1)}$ Médias seguidas de letras iguais, minúsculas nas colunas e maiúsculas nas linhas, para cada interação, não diferem pelo teste de Tukey, a 5\% de probabilidade. (2)Posição 1, entre duas plantas adjacentes, no mesmo alinhamento dos caules na linha de plantio, na "metade" do espaçamento entre plantas, ao se considerar que o espaçamento entre plantas variou de acordo com o tratamento; e posições 2 e 3 , afastadas a 0,25 e 0,50 m do caule, respectivamente, no sentido perpendicular à linha de plantio. 


\section{Conclusões}

1. A cultivar Tupi RN IAC 1669-13 apresenta sistema radicular quantitativa e qualitativamente superior ao da cultivar Catuaí Vermelho IAC 144, para todos os arranjos testados.

2. A redução no espaçamento entre plantas na linha promove aumento em massa de matéria seca, comprimento, volume e área superficial total de raízes por volume de solo, sem alterar o comprimento e a superfície específica de raízes, nem aprofundar o sistema radicular.

3. Os sistemas radiculares das cultivares Catuaí Vermelho IAC 144, Catuaí Amarelo IAC 32 e IAC 62 e Tupi RN IAC 1699-13 são mais abundantes e apresentam atributos morfológicos superiores quando amostrados entre duas plantas adjacentes na linha de plantio, seguidas das posições afastadas a $0,25 \mathrm{~m}$ e $0,50 \mathrm{~m}$ do tronco, com resposta dependente do espaçamento.

4. Há maior abundância de raízes na camada de $0,1 \mathrm{~m}$ de profundidade em comparação à de $0,4 \mathrm{~m}$, para todos os espaçamentos, mas, nesta última profundidade, o sistema radicular da lavoura jovem está em pleno crescimento.

\section{Agradecimentos}

À Fundação de Amparo à Pesquisa do Estado de Minas Gerais (Fapemig), pelo apoio financeiro (processo no CRA-APQ-00044-11); ao Conselho Nacional de Desenvolvimento Científico e Tecnológico $(\mathrm{CNPq})$ e à Coordenação de Aperfeiçoamento de Pessoal de Nível Superior (Capes), pela concessão de bolsas; e à Fazenda Transagro S.A., pela disponibilização da área experimental.

\section{Referências}

ACOMPANHAMENTO DA SAFRA BRASILEIRA: café: safra 2015 - primeiro levantamento - janeiro/2015. Brasília: Conab, v.1, n.1, janeiro 2015. 61p. Disponível em: <http://www.conab.gov.br/ OlalaCMS/uploads/arquivos/15_01_14_11_57_33_boletim_cafe_ janeiro_2015.pdf $>$. Acesso em: 18 jan. 2015.

ALFONSI, E.L.; FAHL, J.I.; CARELLI, M.L.C.; FAZUOLI, L.C. Crescimento, fotossíntese e composição mineral em genótipos de Coffea com potencial parautilização comoporta-enxerto. Bragantia, v.64, p.1-13, 2005. DOI: 10.1590/S0006-87052005000100001.

ALVES, J.D.; PAGLIS, C.M.; LIVRAMENTO, D.E. do; LINHARES, S.S.D.; BECKER, F.B.; MESQUITA, A.C. Source-sink manipulations in Coffea arabica L. and its effect on growth of shoots and root system. Ciência e Agrotecnologia, v.35, p.956-964, 2011. DOI: 10.1590/S1413-70542011000500013.

AMARAL, J.F.T. do; MARTINEZ, H.E.P.; LAVIOLA, B.G.; FERNANDES FILHO, E.I.; CRUZ, C.D. Eficiência de utilização de nutrientes por cultivares de cafeeiro. Ciência Rural, v.41, p.621-629, 2011. DOI: $10.1590 / \mathrm{S} 0103-84782011005000027$.

ANDRADE, A.S.; LIMA, D.T. de; QUEIROZ, V.T. de; DRUMOND, L.C.D. Avaliação da mobilidade potencial de pesticidas em um Latossolo Vermelho-Amarelo. Cerrado Agrociências, v.2, p.76-83, 2011.

ANDRADE, W.E. de B.; GUIMARÃES, P.T.G.; FAQUIN, V.; GUIMARÃES, R.J. Produtividade do cafeeiro arábica em condições de adensamento, no Noroeste Fluminense. Coffee Science, v.9, p.90-101, 2014.

AUGUSTO, H.S.; MARTINEZ, H.E.P.; SAMPAIO, N.F.; CRUZ, C.D.; PEDROSA, A.W. Concentração foliar de nutrientes em cultivares de Coffea arabica L. sob espaçamentos adensados. Ciência e Agrotecnologia, v.31, p.973-981, 2007. DOI: 10.1590/ S1413-70542007000400005.

BARRETO, C.V.G.; SAKAI, E.; ARRUDA, F.B.; SILVA, E.A. da; PIRES, R.C. de M. Distribuição espacial do sistema radicular do cafeeiro fertirrigado por gotejamento em Campinas. Bragantia, v.65, p.641-647, 2006. DOI: 10.1590/S0006-87052006000400015.

BLISKA, F.M.M.; FAZUOLI, L.C.; BRAGHINI, M.T. Impactos de cultivares resistentes a doenças e pragas no desenvolvimento sustentável da cafeicultura nas principais regiões cafeeiras do brasil. In: SIMPÓSIO DE PESQUISA DOS CAFÉS DO BRASIL, 7., 2011, Araxá. Anais. Araxá: EMBRAPA-CAFÉ, 2011. 7p.

BRACCINI, A. de L.E. e; SCAPIM, C.A.; VIDIGAL FILHO, P.S.; BRACCINI, M. do C.L. BORGES, S.C.; ALBRECHT, L.P. Características agronômicas e produção de frutos e grãos em resposta ao aumento na densidade populacional do cafeeiro. Acta Scientiarum. Agronomy, v.27, p.269-279, 2005. DOI: 10.4025/ actasciagron.v27i2.1845.

BRAGANÇA, S.M. Crescimento e acúmulo de nutrientes pelo cafeeiro conilon (Coffea canephora Pierre). 2005. 99p. Tese (Doutorado em Fitotecnia) - Universidade Federal de Viçosa, Viçosa.

BRAGANÇA, S.M.; SILVA, E.B.; GUARÇONI M., A.; SANTOS, L.P.; LANI, J.A.; VOLPI, P.S. Resposta do cafeeiro conilon à adubação de NPK em sistema plantio adensado. Coffee Science, v.4, p.67-75, 2009.

CARVALHO, C.H.S. de. (Ed.). Cultivares de café: origem, características e recomendações. Brasília: Embrapa Café, 2008. $334 \mathrm{p}$.

CASSIDY, D.M.S.; KUMAR, D. Root distribution of Coffea arabica L. in Zimbabwe. I. The effect of plant density, mulch, cova planting and shade in Chipinge. Zimbabwe Journal of Agricultural Research, v.22, p.119-132, 1984.

COVRE, A.M.; PARTELLI, F.L.; MAURI, A.L.; DIAS, M.A. Crescimento e desenvolvimento inicial de genótipos de café conilon. Revista Agro@mbiente On-line, v.7, p.193-202, 2013.

DAMATTA, F.M.; RONCHI, C.P.; MAESTRI, M.; BARROS, R.S. Ecophysiology of coffee growth and production. Brazilian 
Journal of Plant Physiology, v.19, p.485-510, 2007. DOI: 10.1590/S1677-04202007000400014.

FERNANDES, A.L.T.; PARTELLI, F.L.; BONOMO, R.; GOLYNSKI, A. A moderna cafeicultura dos cerrados brasileiros. Pesquisa Agropecuária Tropical, v.42, p.231-240, 2012. DOI: 10.1590/S1983-40632012000200015.

FIGUEIREDO, F.C.; FURTINI NETO, A.E.; GUIMARÃES, P.T.G.; SILVA, E. de B.; BOTREL, P.P. Eficiência da adubação com NPK na produção de cafezais adensados na Região Sul de Minas Gerais. Coffee Science, v.1, p.135-142, 2006.

GUARÇONI M., A. Características da fertilidade do solo influenciadas pelo plantio adensado de café conilon. Semina: Ciências Agrárias, v.32, p.949-958, 2011. DOI: 10.5433/1679-0359.2011v32n3p949.

JORGE, L.A. de C.; RODRIGUES, A.F. de O. Safira: sistema de análise de fibras e raízes. São Carlos: Embrapa Instrumentação Agropecuária, 2008. 21p. (Embrapa Instrumentação Agropecuária. Boletim de pesquisa e desenvolvimento, 24).

MATIELLO, J.B.; SANTINATO, R.; GARCIA, A.W.R.; ALMEIDA, S.R.; FERNANDES, D.R. Cultura de café no Brasil: manual de recomendações. Rio de Janeiro: MAPA/Procafé; Varginha: Fundação Procafé, 2010. 546p.

MOTA, A.C.V.; NICK, J.A.; YORINORI, G.T.; SERRAT, B.M. Distribuição horizontal e vertical da fertilidade do solo e das raízes de cafeeiro (Coffea arabica L.) cultivar Catuaí. Acta Scientiarum. Agronomy, v.28, p.455-463, 2006. DOI: 10.4025/actasciagron. v28i4.758.

MOTTA, P.E.F. da; BARUQUI, A.M.; SANTOS, H.G. dos. Levantamento de reconhecimento de média intensidade dos solos da região do alto Paranaíba, Minas Gerais. Rio de Janeiro: Embrapa Solos, 2004. 414p. (Embrapa Solos. Boletim de pesquisa e desenvolvimento, 44).

PARTELLI, F.L.; COVRE, A.M.; OLIVEIRA, M.G.; ALEXANDRE, R.S.; VITÓRIA, E.L. da; SILVA, M.B. da. Root system distribution and yield of 'Conilon' coffee propagated by seeds or cuttings. Pesquisa Agropecuária Brasileira, v.49, p.349-355, 2014. DOI: 10.1590/S0100-204X2014000500004.

PARTELLI, F.L.; VIEIRA, H.D.; SANTIAGO, A.R.; BARROSO, D.G. Produção e desenvolvimento radicular de plantas de café 'Conilon' propagadas por sementes e por estacas. Pesquisa Agropecuária Brasileira, v.41, p.949-954, 2006. DOI: 10.1590/ S0100-204X2006000600008.

PAULO, E.M.; FURLANI JUNIOR, E.; FAZUOLI, L.C. Comportamento de cultivares de cafeeiro em diferentes densidades de plantio. Bragantia, v.64, p.397-409, 2005. DOI: 10.1590/ S0006-87052005000300009.
PAVAN, M.A.; CHAVES, J.C.D.; ANDROCIOLI FILHO, A. Produção de café em função da densidade de plantio, adubação e tratamento fitossanitário. Turrialba, v.44, p.227-231, 1994.

PAVAN, M.A.; CHAVES, J.C.D.; SIQUEIRA, R.; ANDROCIOLI FILHO, A.; COLOZZI FILHO, A.; BALOTA, E.L. High coffee population density to improve fertility of an oxisol. Pesquisa Agropecuária Brasileira, v.34, p.459-465, 1999. DOI: 10.1590/ S0100-204X1999000300018.

PEREIRA, S.P.; BARTHOLO, G.F.; BALIZA, D.P.; SOBREIRA, F.M.; GUIMARÃES, R.J. Crescimento, produtividade e bienalidade do cafeeiro em função do espaçamento de cultivo. Pesquisa Agropecuária Brasileira, v.46, p.152-160, 2011. DOI: 10.1590/S0100-204X2011000200006.

PINHEIRO, H.A.; DAMATTA, F.M.; CHAVES, A.R.M; LOUREIRO, M.E.; DUCATTI, C. Drought tolerance is associated with rooting depth and stomatal control of water use in clones of Coffea canephora. Annals of Botany, v.96, p.101-108, 2005. DOI: 10.1093/aob/mci154.

RENA, A.B.; DAMATTA, F.M. O sistema radicular do cafeeiro: estrutura e ecofisiologia. In: ZAMBOLIN, L. (Ed.). O estado da arte de tecnologias na produção de café. Viçosa: UFV, 2002. p.11-92.

RENA,A.B.; GUIMARÃES, P.T.G. Sistema radicular do cafeeiro: estrutura, distribuição, atividade e fatores que o influenciam. Belo Horizonte: Epamig, 2000. 80p. (EPAMIG. Documentos, 37).

RODRIGUES, L.A.; MARTINEZ, H.E.P.; NEVES, J.C.L.; NOVAIS, R.F.; MENDONÇA, S.M. Growth response of coffee tree shoots and roots to subsurface liming. Plant and Soil, v.234, p.207-214, 2001. DOI: 10.1023/A:1017999318532.

RONCHI, C.P.; TERRA, A.A.; SILVA, A.A. Growth and nutrient concentration in coffee root system under weed species competition. Planta Daninha, v.25, p.679-687, 2007. DOI: 10.1590/S0100-83582007000400004.

SANTINATO, R.; FERNANDS, A.L.T.; FERNANDES, D.R. Irrigação na cultura do café. 2.ed. Belo Horizonte: $O$ Lutador, 2008. 476p.

SILVA, A.L. da; BRUNO, I.B.; REICHARDT, K.; BACCHI, O.O.S.; DOURADO-NETO, D.; FAVARIN, J.L.; COSTA, F.M.P. da; TIMM, L.C. Soil water extraction by roots and $\mathrm{Kc}$ for the coffee crop. Revista Brasileira de Engenharia Agrícola e Ambiental, v.13, p.257-261, 2009. DOI: 10.1590/S1415-43662009000300006.

SILVA, V.A.; ANTUNES, W.C.; GUIMARÃES, B.L.S.; PAIVA, R.M.C.; SILVA, V. de F.; FERRÃO, M.A.G.; DAMATTA, F.M.; LOUREIRO, M.E. Resposta fisiológica de clone de café Conilon sensível à deficiência hídrica enxertado em porta-enxerto tolerante. Pesquisa Agropecuária Brasileira, v.45, p.457-464, 2010. DOI: 10.1590/S0100-204X2010000500004. 